\title{
Os impactos na capacidade atencional em trabalhadores usuários de drogas
}

\author{
The impacts of drug use on worker's attention capacity
}

\author{
Oliveira Alves de Lima Junior', Fernanda Mayrink Gonçalves², Renato Wilson Santos Cabral', \\ Lizânia Spinassé Borges ${ }^{3}$, Cesar Albenes de Mendonça Cruz', Hebert Wilson Santos Cabral ${ }^{1,2}$
}

\begin{abstract}
RESUMO | Contexto: $\mathrm{O}$ uso de drogas tem tomado grandes dimensões e repercute na saúde pública e ambiente de trabalho, expondo o indivíduo a um maior risco de acidentes de trabalho e ao comprometimento de sua qualidade de vida. Objetivo: Comparar o desempenho da atenção de empregados usuários de drogas e não usuários, em exame computadorizado de atenção (EAC). Métodos: Trata-se de um estudo retrospectivo que avaliou o desempenho dos indivíduos no EAC em cada um dos seus parâmetros ("omissão", " erros", "média do tempo de reação" e "variabilidade do tempo de reação"). Nas análises, foram utilizadas a descritiva, o Teste t, o Teste de Mann-Whitney e as correlações múltiplas. Resultados: Foram selecionados 23 controles (PC) e 23 dependentes químicos (DQ), dos quais 9 faziam uso de 3 tipos de drogas ou mais, sendo que a substância prevalente foi o álcool (100,00\%) e a associação mais frequente foi deste com a cocaína (69,56\%). No que tange ao EAC, os valores obtidos de média \pm desvio padrão

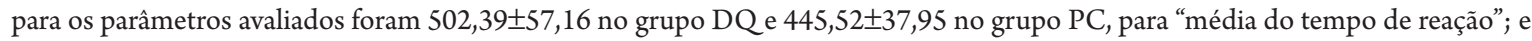
$92,96 \pm 26,48$ no grupo DQ e 66,26 $\pm 11,86$ no grupo PC, para “variabilidade no tempo de reação". Os valores de mediana e erro padrão

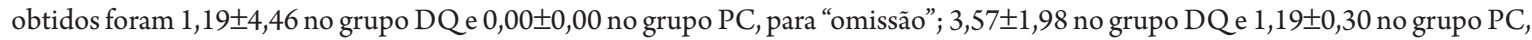
para "erros". Conclusão: Observou-se a prevalência do uso de álcool, principalmente em associação com cocaína. Sobre a atenção, os resultados demonstraram que, independente do parâmetro, todos os valores são inferiores aos encontrados em controles normais $(\mathrm{p}<0,05)$, demonstrando que o uso de qualquer tipo de droga compromete o desempenho atencional, podendo limitar atividades laborais e gerar impacto nas atividades de vida diária, colocando em risco a saúde e segurança dos trabalhadores.
\end{abstract}

Palavras-chave I atenção; testes neuropsicológicos; saúde do trabalhador; transtornos relacionados ao uso de substâncias.

\begin{abstract}
Context: The use of drugs has large effects in public health and the working environment, potentially exposing workers to the risk of accidents and compromising their quality of life. Objectives: To compare the attention span between drug users and normal employees (controls), using a computerized test of their attention. Methods: The design of this study was retrospective, evaluating the performance of individuals with a test in each of its parameters ("omission", "errors", "average reaction time" and "variability of reaction time").Thus, the descriptive analysis included $t$ and Mann-Whitney tests and multiple correlations. Results: The current study selected a total of 23 controls (PC) and 23 drug users (DQ), of which 9 were using 3 or more different types of drugs; the most prevalent substance was alcohol (100.00\%) and the most frequent association was alcohol and cocaine (69.56\%). Based on the computerized attention test, the values of mean \pm standard deviation were $502.39 \pm 57.16$ for the chemically dependent group and $445.52 \pm 37.95$ for the control group to "average reaction time"; $92.96 \pm 26.48$ for the chemically dependent group and $66.26 \pm 11.86$ for the control group to "variability in reaction time". The median and standard error values were $1.19 \pm 4.46$ for the chemically dependent group and $0.00 \pm 0.00$ for the control group to "omissions"; $3.57 \pm 1.98$ for the chemically dependent group and $1.19 \pm 0.30$ for the control group to "errors". Conclusion: It was possible to observe a higher prevalence of alcohol abuse especially in combination with cocaine. The results showed that regardless of the parameters all the result scores are lower than those found in normal controls $(p<0.05)$, showing that the use of any type of drug may influence attentional performance, restrict labor activities and impact other daily activities resulting in damage to the health and safety of employees.
\end{abstract}

Keywords I attention; neuropsychological tests; occupational health; substance-related disorders.

Trabalho realizado na Escola Superior de Ciências da Santa Casa de Misericórdia de Vitória (EMESCAM) - Bela Vista (ES), Brasil 'Centro de Ciências da Saúde de Vitória, EMESCAM - Vitória (ES), Brasil.

2Programa de Pós-graduação em Neurologia/Neurociências da Universidade Federal Fluminense (UFF) - Niterói (RJ), Brasil.

3Universidade Paris XI - Paris, França.

DOI: $10.5327 / Z 1679-443520160915$ 


\section{INTRODUÇÃO}

O consumo de drogas, tanto lícitas quanto ilícitas, vem se expandido globalmente. As drogas representam uma das questões que afligem a sociedade contemporânea, sendo que, em escala mundial, constituem uma ameaça às estruturas e valores econômicos, políticos, sociais e culturais das nações. A Organização Mundial de Saúde conceitua droga como "qualquer entidade química ou mistura de entidades (mas outras que não aquelas necessárias à manutenção da saúde, como água e o oxigênio) que altera a função biológica e possivelmente sua estrutura"1. A esse conceito, estudos ${ }^{2-5}$ acrescentam que tal alteração afeta os indivíduos nos seus níveis físico, social e cognitivo.

No século XX, a partir da década de 1960, o consumo de drogas foi mais intensificado e se tornou uma questão de saúde pública. Algumas práticas contribuíram para incrementar o uso dos chamados psicotrópicos - drogas que agem no sistema nervoso central (SNC) —, principalmente a síntese de novos produtos aumentando a concentração e a potência das drogas tradicionais, possibilitando ao indivíduo obter efeitos diversificados ${ }^{6,7}$.

Dentro do conceito de promoção da saúde na área do trabalhador encontra-se a análise dos hábitos de vida do indivíduo e o acompanhamento do estado geral da saúde ${ }^{8}$, que engloba o uso de drogas ${ }^{9}$. Atualmente, existem várias ferramentas desenvolvidas para auxiliar no tratamento do usuário de droga, porém é preciso evitar metas utópicas de erradicação total do abuso, como provam as evidências históricas e antropológicas. As novas metodologias podem dar indicativos de alterações comuns encontradas em indivíduos com dependência química ${ }^{10}$.

Ao considerar o mercado de trabalho, o imediatismo, os medos, a pressão por incertezas e frustrações são características da sociedade contemporânea. Essas características frequentemente expõem o funcionário a situações de riscos físico e psíquico, com o aumento dos transtornos mentais em trabalhadores, levando-os ao consumo de drogas, expondo-os a acidentes de trabalho, à incapacidade para o trabalho, ao afastamento do trabalho por tempo prolongado e à exclusão do mercado de trabalho, aumentando os custos para o Estado e a sociedade ${ }^{9,11}$.

Vários estudos demonstram que o uso de drogas acarreta alteração da atenção e memória, o que, em se tratando de saúde do trabalhador, coloca em risco sua atividade, segurança e qualidade de vida. Sendo assim, o presente trabalho teve por objetivo comparar o desempenho da atenção em empregados não dependentes químicos e empregados usuários de drogas, por meio de exame computadorizado.

\section{MÉTODOS}

\section{SELEÇÃO DA AMOSTRA}

Trata-se de um estudo retrospectivo que avaliou o desempenho atencional de empregados dependentes químicos (DQ) que buscaram voluntariamente, em um Centro Especializado, tratamento para o abuso de drogas, no período de setembro de 2011 a maio de 2012, perfazendo um grupo de 23 indivíduos.

Foram obtidos os dados sociodemográficos (idade e gênero), o tipo de drogas utilizadas (álcool, nicotina, maconha, cocaína ou crack) e o resultado do exame computadorizado de atenção (EAC) no que se refere às quatro variáveis principais: "omissão", "erros", "média do tempo de reação" e "variabilidade do tempo de reação".

O grupo controle (PC) foi composto por 23 indivíduos não dependentes químicos. Para obtenção dos sujeitos controles, foram selecionados aleatoriamente indivíduos pareados por perfil sociodemográfico e características clínicas sem déficit de atenção.

Os critérios de inclusão foram idade menor que 60 anos, ausência de uso de medicamentos benzodiazepínicos, hipnóticos, sedativos, inibidores da Mao, inibidores seletivos ou não da recapitação de serotonina, bem como não uso único e exclusivo de nicotina, problemas significativos no nascimento ou desenvolvimento psicomotor, outros diagnósticos psiquiátricos do eixo I do Diagnostic and Statistical Manual of Mental Disorders (DSM-IV), sem história de traumatismo cranioencefálico ou problemas neurológicos, assim como ausência de problemas clínicos evidenciados no SNC.

O presente estudo foi aprovado pelo Comitê de Ética e Pesquisa da Escola Superior de Ciências da Santa Casa de Misericórdia de Vitória (EMESCAM), sob o Certificado de Apresentação para Apreciação Ética (CAAE) $n^{\circ} 24154813.8 .0000 .5065$.

\section{INSTRUMENTOS}

Os instrumentos utilizados foram os prontuários físicos e eletrônicos que continham os dados sociodemográficos e 
as informações sobre a utilização de substâncias psicoativas supracitadas, bem como os resultados do EAC.

O EAC foi desenvolvido por Cabral e Cabral ${ }^{12}$ com base no continuous performance test $(\mathrm{CPT})^{3,13,14}$. Trata-se de um instrumento computadorizado de rastreio da atenção que mensura variáveis atencionais. $\mathrm{O}$ teste consiste no aparecimento de vários símbolos na tela, em diversas posições e velocidade variável. Ao início de cada exame, o programa aponta qual símbolo será considerado correto e a partir de então o indivíduo precisa apertar a tecla "espaço" do teclado do computador toda vez que esse símbolo aparecer. Em seguida, é apresentado na tela do computador um questionário pós-teste com perguntas referentes a queixas de saúde mental, problemas financeiros, familiares e no trabalho. Existem diferentes versões do EAC: rastreio de 1,5 minuto e concentrada 5,5/11/16 minutos. Foi utilizada a versão concentrada de 11 minutos. $\mathrm{O}$ teste mensura porcentagem de acertos, porcentagem de erros, porcentagem de omissões, tempo médio de reação de acertos e erros, variabilidade do tempo de reação de acertos e erros, os quais fornecem as informações sobre mensuração dos índices de omissão (refletindo a desatenção), erros (refletindo a impulsividade), tempo de reação e distração.

\section{ANÁLISE ESTATÍSTICA}

Foram analisadas as descritivas das variáveis sociodemográficas dos grupos DQ e PC. As variáveis do EAC ("omissão", "erros", "média do tempo de reação" e "variabilidade do tempo de reação") foram testadas quanto à distribuição ou não de normalidade pelo teste KolmogorovSmirnov (KS). Os parâmetros "omissão" e "erros" não apresentaram distribuição normal, sendo trabalhados com o teste Mann-Whitney. Os parâmetros "média do tempo de reação" e "variabilidade do tempo de reação" apresentaram distribuição normal e foram trabalhados por meio do teste $t$. Nas comparações, foram utilizados: teste qui-quadrado, teste te Mann-Whitney. Foi adotado o erro $\beta$ de $20 \%$ e o $\alpha$ de $5 \%$.

\section{RESULTADOS}

A amostra foi constituída por 46 indivíduos, dos quais 23 no grupo PC e 23 no grupo DQ.

Quanto ao tipo de substância utilizada, observou-se a prevalência do uso do álcool (100,00\%) quando comparado com outras drogas, tendo a cocaína $(69,56 \%)$ como a segunda mais utilizada. Constatou-se, ainda, o uso de maconha, nicotina e crack.

Dos indivíduos avaliados, 69,56\% faziam uso de pelo menos 2 substâncias psicoativas, sendo que houve associação mais frequente $(69,56 \%)$ entre o álcool e a cocaína.

A comparação do desempenho nas variáveis no exame EAC está representada na Tabela 1.

\section{DISCUSSÃO}

O estudo mostrou que, em uma amostra de empregados adultos jovens, predominantemente do sexo masculino e usuários de drogas, as substâncias mais utilizadas são o álcool e a cocaína, sendo comum a associação de mais de uma substância psicoativa.

Esses achados vão ao encontro do relatado em literatura por um levantamento do Centro Brasileiro de Informações sobre Drogas Psicotrópicas (CEBRID), realizado em 2004, sobre internações em hospitais psiquiátricos por uso de substâncias. Esse estudo demonstrou que o álcool é a primeira causa de internação e a cocaína é a terceira, sendo o uso de multidrogas - isto é, o uso concomitante de mais de um tipo de droga - a segunda causa de internação ${ }^{15}$.

Tabela 1. Comparação das médias de "omissão" e "erros" e medianas de "média do tempo de reação" e "variabilidade do tempo de reação" dos pacientes dos grupos de dependentes químicos e controle.

\begin{tabular}{|c|c|c|c|c|c|}
\hline & \multicolumn{2}{|c|}{$D Q(n=23)$} & \multicolumn{2}{|c|}{$P C(n=23)$} & \multirow[b]{2}{*}{ Sig. } \\
\hline & $\begin{array}{c}\text { Mediana*/ } \\
\text { Média** }^{*}\end{array}$ & $\begin{array}{c}\text { Erro } \\
\text { Padrão*/ } \\
\text { DVP }^{* *}\end{array}$ & $\begin{array}{c}\text { Mediana*/ } \\
\text { Média** }^{*}\end{array}$ & $\begin{array}{c}\text { Erro } \\
\text { Padrão*/ } \\
\text { DVP** }^{*}\end{array}$ & \\
\hline Omissão* & 1,19 & 4,46 & 0,00 & 0,00 & 0,000 \\
\hline Erros* $^{*}$ & 3,57 & 1,98 & 1,19 & 0,30 & 0,001 \\
\hline $\begin{array}{l}\text { Média do } \\
\text { tempo de } \\
\text { reação*^ }\end{array}$ & 502,30 & 57,15 & 445,50 & 37,94 & 0,003 \\
\hline $\begin{array}{l}\text { Variabilidade } \\
\text { do tempo de } \\
\text { reação** }\end{array}$ & 92,90 & 26,47 & 66,20 & 11,85 & 0,001 \\
\hline
\end{tabular}

*Mann-Whitney; **Teste t de Student; DQ: grupo de dependentes químicos; PC: grupo controle; DVP: desvio padrão; Sig.: significância. 
O álcool é reconhecido como a substância psicoativa mais utilizada no mundo, apresentando alta prevalência também no Brasil ${ }^{16-19}$. Além disso, o uso abusivo de álcool está associado a altos índices de consumo de substâncias ilícitas, além de ser frequente a utilização de multidrogas, como observado no presente trabalho ${ }^{17}$.

Este estudo observou o comprometimento de todas as variáveis atencionais avaliadas, sendo elas: "omissão" (desatenção), "erros" (impulsividade), "média do tempo de reação" (velocidade de reação motora) e "variabilidade do tempo de reação" (concentração).

Prosser, London e Galynker ${ }^{3}$ avaliaram o desempenho atencional, por meio de CPT, em indivíduos usuários de multidrogas e em abstinência, que já fizeram (grupo 1) ou mantêm tratamento com metadona (grupo 2), comparando-os com um grupo controle (grupo 3 ). Esses pesquisadores observaram um pior desempenho dos grupos de ex-usuários (grupos 1 e 2) nos parâmetros "erros" e "omissão", corroborando com os achados do deste trabalho.

O comprometimento atencional foi observado também por Hanson et al..$^{20}$, que avaliaram o desempenho cognitivo de adolescentes usuários de álcool e de outras drogas por um período de dez anos. Destacaram o comprometimento da atenção principalmente naqueles jovens que aumentaram a dosagem de consumo no final da adolescência ou início da fase adulta, quando comparados com indivíduos controles. Da mesma forma, descreveram que os achados sugerem que os jovens que interromperam o uso durante a adolescência apresentaram desempenho semelhante àqueles que não faziam uso, porém a continuidade no uso com altas doses durante o final do período da adolescência ou no início da fase adulta representou um aumento do impacto na cognição, mesmo após a redução do consumo.

A alteração da atenção em usuários de drogas também foi observada no estudo de Indlekofer et al. ${ }^{21}$, sugerindo uma forte tendência a lapsos de atenção; porém não observou diferenças na média do tempo de reação quando comparados ao grupo controle, o que difere dos presentes achados.

A piora na média do tempo de reação observada neste estudo pode ser representativa e justificada pela lentificação da reação motora em indivíduos em abstinência ${ }^{22}$, porém pode ainda ser em virtude do pequeno tamanho da amostra, sendo, assim, sugerida realização de novos estudos com amostragem maior.

O comprometimento cognitivo em usuários de drogas é foco de vários trabalhos, e nesse quesito surgem alguns fatores e questionamentos que possivelmente interferem no grau de comprometimento cognitivo, os quais geralmente não são controlados e dificultam a comparação entre os diversos estudos existentes. São alguns desses fatos de questionamentos: controle da droga utilizada ${ }^{23-25}$; tempo de uso ${ }^{25-27}$; idade de início de consumo ${ }^{25,26}$; tempo de abstinência ${ }^{24,25}$; doses ${ }^{25,26}$; e condições sociodemográficas ${ }^{27}-$ não controladas no presente estudo e que devem ser foco dos futuros trabalhos.

\section{CONCLUSÕES}

O presente estudo observou um pior desempenho de empregados dependentes químicos no teste de atenção quando comparados a controles normais, apontando para uma alteração nesse domínio cognitivo independente dos parâmetros atencionais avaliados. Essa condição pode limitar as atividades laborais dos indivíduos, levando-os a risco de acidentes e comprometendo sua saúde. Os testes computacionais vêm com objetivo de contribuir para uma melhor qualidade de vida desses empregadores, por estar voltado diretamente para as mudanças do comportamento individual. Dentro de uma grande empresa, por exemplo, o uso desse método auxilia na diminuição dos riscos de acidentes de trabalho.

\section{REFERÊNCIAS}

1. World Health Organization. Community response to alcohol-related problems. Geneva: World Health Organization; 1985.

2. Cunha PJ, Nicastri S, Gomes LP, Moino RM, Peluso MA. Alterações neuropsicológicas em dependentes de cocaína/ crack internados: dados preliminares. Rev Bras Psiquiatr. 2004:26(2):103-6.
3. Prosser J, London ED, Galynker II. Sustained attention in patients receiving and abstinent following methadone maintenance treatment for opiate dependence: performance and neuroimaging results. Drug Alcohol Depend. 2009;104(3):228-40.

4. Corrêa Filho JM, BaltieriDA. Psychosocial and clinical predictors of retention in outpatient alcoholism treatment. Rev Bras Psiquiatr.2012;34(4):413-21. 
5. Anderson KG, Ramo DE, Cummins KM, Brown SA. Alcohol and drug involvement after adolescent treatment and functioning during emerging adulthood. Drug Alcohol Depend. 2010;107(2-3):171-81.

6. Abramovay M, Castro MG, Pinheiro LC, Lima FS, Martinelli CC. Juventude, violência e vulnerabilidade social na América Latina: desafios para políticas públicas. Brasília: Unesco, Banco Interamericano de Desenvolvimento; 2002.

7. Castro MG, Abramovay M, Rua MG, Andrade ER. Cultivando vida, desarmando violências: experiências em educação, cultura, lazer, esporte e cidadania com jovens em situações de pobreza. Brasília: UNESCO, Brasil Telecom, Fundação Kellogg, Banco Interamericano de Desenvolvimento; 2001

8. Teixeira CG, Gonçalves FM, Cabanas RC, Rigoni GS, Cabral HW. Avaliação de preditores de risco de comprometimento clínico em empregados de empresa mineradora: contribuições para qualidade de vida. Rev Bras Med Trab. 2014;12(1):30-8.

9. Sousa-Uva A, Serranheira F. Trabalho e Saúde/(Doença): o desafio sistemático da prevenção dos riscos profissionais e o esquecimento reiterado da promoção da saúde. Rev Bras Med Trab. 2013;11(1):43-9.

10. Babor T, Caetano R, Casswell S, Edward G, Giesbrecht N, Graham, K, et al. Alcohol: no ordinary commodity: research and public policy. Oxford University Press; 2003.

11. Seligmann-Silva E, Bernanrdo MH, Maeno M, Kato M. O mundo contemporâneo do trabalho e a saúde mental do trabalhador. Rev Bras Saúde Ocup. 2010;35(122):187-91.

12. Cabral HWS, Cabral RWS. Exame Computadorizado de Atenção EAC. 2006.

13. Bartés-Serrallonga M, Adan A, Solé-Casals J, Caldú X, Falcón C, PérezPàmies M, Bargalló N, Serra-Grabulosa JM. Cerebral networks of sustained attention and working memory: a functional magnetic resonance imaging study based on the Continuous Performance Test. Rev Neurol. 2014;58(7):289-95.

14. Rapisarda A, Kraus M, Tan YW, Lam M, Eng GK, Lee J, et al. The continuous performance test, identical pairs: norms, reliability and performance in healthy controls and patients with schizophrenia in Singapore. Schizophr Res. 2014;156(2-3):233-40.

15. Carlini EA. Epidemiologia do uso de álcool no Brasil. Arq Med ABC. 2006;31(Suppl. 2):4-7.
16. Kolling NM, Silva CR, Carvalho JC, Cunha SM, Kristensen $\mathrm{CH}$. Avaliação neuropsicológica em alcoolistas e dependentes de cocaína. Aval Psicol. 2007;6(2):127-37.

17. Harrington M, Velicer WF, Ramsey S. Typology of alcohol users based on longitudinal patterns of drinking. Addict Behav. 2014;39(3):607-21.

18. Vieira RM, Serafim AP, Saffi F. Prejuízos neurocognitivos na dependência alcoólica: um estudo de caso. Rev Psiquiatr Clín. 2007;34(5);246-50.

19. Sousa FS, Oliveira EN. Caracterização das internações de dependentes químicos em Unidade de Internação Psiquiátrica do Hospital Geral. Ciênc Saúde Coletiva. 2010;15(3):671-7.

20. Hanson KL, Cummins K, Tapert SF, Brown SA. Changes in neuropsychological functioning over 10 years following adolescent substance abuse treatment. Psychol Addict Behav. 2011;25(1):127-42.

21. Indlekofer F, Piechatzek M, Daamen M, Glasmacher C, Lieb R, Pfister $\mathrm{H}$, et al. Reduced memory and attention performance in a population-based sample of young adults with a moderate lifetime use of cannabis, ecstasy and alcohol. J Psychopharmacol. 2009;23(5):495-509.

22. Sadock BJ, Sadock VA. Compêndio de Psiquiatria: ciência do comportamento e psiquiatria clínica. 9. ed. Porto Alegre: Artmed;2007.

23. Gould TJ. Addiction and Cognition. AddictSci Clin Pract. 2010;5(2):4-14.

24. Fernández-Serrano MJ, Pérez-García M, Verdejo-García A. What are the specific vs. generalized effects of drugs of abuse on neuropsychological performance? Neurosci Biobehav Rev. 2011;35(3):377-406

25. Thames AD, Arbid N, Sayegh P. Cannabis use and neurocognitive functioning in a non-clinical sample of users. Addict Behav. 2014;39(5):994-9.

26. Sinforiani E, Zucchella C, Pasotti C, Casoni F, Bini P, Costa A. The effects of alcohol on cognition in the elderly: from protection to neurodegeneration. Funct Neurol. 2011;26(2):103-6.

27. Loeber S, Nakovics H, Kniest A, Kiefer F, Mann K, Croissant B. Factors affecting cognitive function of opiate-dependent patients. Drug Alcohol Depend. 2012;120(1-3):81-7.

Endereço para correspondência: Fernanda Mayrink Gonçalves - Rua Doutor Moacyr Gonçalves, 101/302 - Jardim da Penha - CEP: 29060-445 - Vitória (ES), Brasil - E-mail: nandamayrink@yahoo.com.br 